EPJ Web of Conferences 84, 07001 (2015)

DOI: $10.1051 /$ epjconf/20158407001

(C) Owned by the authors, published by EDP Sciences, 2015

\title{
Theory of dissociative electron attachment: Biomolecules and clusters
}

\author{
Ilya I. Fabrikant ${ }^{\mathrm{a}}$ \\ Department of Physics and Astronomy, University of Nebraska, Lincoln, NE 68588, USA
}

\begin{abstract}
Very broad $\sigma^{*}$ resonances, which are responsible for threshold structures and dissociative attachment in electron collisions with hydrogen halides, are also important in electron-impact bond-breaking in nucleobases and amino acids. We investigate this mechanism in more detail by carrying out model calculations of the $\mathrm{N}-\mathrm{H}$ bond breaking in the uracil molecule. Although the $\sigma^{*}$ resonance is extremely broad at the equilibrium nuclear geometry, it is stabilized fast when the $\mathrm{N}-\mathrm{H}$ bond is stretched, and this produces a substantial dissociative attachment cross section. In addition, very pronounced vibrational Feshbach resonances are seen below vibrational excitation thresholds. To incorporate the effect of a cluster environment in the dissociative electron attachment process, we develop further the multiple scattering theory for this process and calculate the dissociative attachment cross section for the $\mathrm{CF}_{3} \mathrm{Cl}$ molecule embedded in the $\left(\mathrm{H}_{2} \mathrm{O}\right)_{6}$ cluster.
\end{abstract}

\section{Introduction}

Interaction of low-energy electrons with DNA nucleobases (thymine, adenine, cytosine, and guanine), organic acids, and amino acids is of great significance for the description of the molecular mechanisms in radiation damage [1-8]. When high-energy radiation interacts with a biological medium, it produces free radicals and low-energy electrons. This is a particularly important process in aqueous solutions as the biological damage induced by free radicals from the radiolysis of water far exceeds that by direct energy deposition to DNA [9]. Whereas the role of free radicals in the radiation damage has been well established, the role of low-energy electrons is still under intense investigations. Low-energy electrons in a medium are eventually trapped in a potential well and become ineffective in inducing biological damage [10]. However, before becoming solvated, they exist in so-called presolvated or precursor states with the lifetime of about 0.2-0.5 ps [11]. These presolvated electrons can very efficiently break bonds in chlorofluorocarbons [12] and DNA [13].

To understand the mechanism for bond breaking in biological molecules by low-energy electrons, we have to consider first their interaction with isolated molecules and investigate the dissociative electron attachment (DEA) processes which are primarily responsible for bond breaking at low energies. Then we have to investigate how these processes are affected by water environment. One way to study the

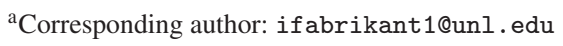

This is an Open Access article distributed under the terms of the Creative Commons Attribution License 4.0, which permits unrestricted use, distribution, and reproduction in any medium, provided the original work is properly cited. 


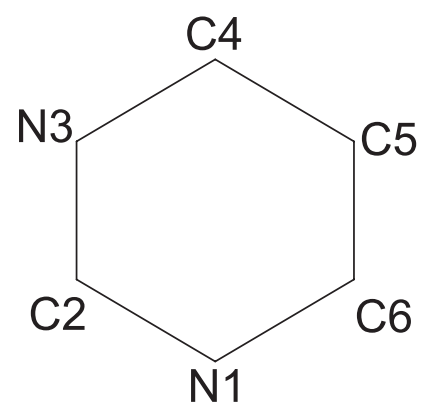

Figure 1. Molecular structure of the uracil and thymine ring. Numbers by the chemical symbols indicate the atom positions. In this paper we discuss the N-H bond breaking at the N1 position.

latter problem theoretically is to place a molecule in an environment of a water cluster. The present paper reviews recent attempts to solve these problems.

With regard to DEA in electron collisions with biological molecules, we will be interested in the process of hydrogen loss, i.e. the reaction

$$
e+M \rightarrow(M-\mathrm{H})^{-}+\mathrm{H}
$$

where $(M-\mathrm{H})^{-}$denotes the closed shell anion formed by the ejection of a neutral hydrogen radical from the temporary negative ion. From gas phase experiments, it was found that all the nucleobases [1-4], organic acids [5] and also amino acids [6-8] exhibit a hydrogen loss due to low-energy electrons. In biological environment, the anion $(M-\mathrm{H})^{-}$can initiate further reactions leading to the cell damage.

To study the effects of a water environment, we place the attaching molecule in a water cluster and use the multiple-scattering theory $[14,15]$ to investigate how the water molecules affect the attachment amplitude and the DEA cross sections.

\section{Hydrogen loss in uracil and thymine}

\subsection{Mechanisms}

According to experimental [16-18] and theoretical [19] studies, the problem of the $(M-\mathrm{H})^{-}$formation, where $M$ stands for uracil or thymine, can be separated into two parts.

The $\mathrm{H}$ loss from the $\mathrm{N} 1$ site (see Fig. 1) can be treated as a direct capture into the lowest $\sigma^{*}$ unoccupied orbital assisted by the interaction with the dipole-supported states. The $\mathrm{H}$ loss from the $\mathrm{N} 3$ site should be treated as capture into the second unoccupied $\pi^{*}$ orbital with the following transition into the second $\sigma^{*}$ state due to vibronic coupling. In the latter case the DEA mechanism is similar to that observed previously for chlorobenzene and vinyl chloride [20]. Although it is hard to separate these two reaction channels in practice, information about the cross section for the first reaction can be provided by performing experiments with uracil or thymine methylated at the $\mathrm{N} 3$ position [21]. Calculation of cross sections for these two channels involves different physics, and therefore requires different methods. The $\mathrm{H}$ loss from the $\mathrm{N} 1$ site involves a broad $\sigma^{*}$ shape resonance and vibrational Feshbach resonances (VFRs) [22], and therefore requires the nonlocal complex potential approach [23], or equivalent R-matrix approach [24]. On the other hand, the H loss from the N3 site is primarily due to the relatively narrow $\pi^{*}$ resonance, and the local approximation is adequate in this case. Unfortunately, the necessary inclusion of the vibronic coupling between $\sigma^{*}$ and $\pi^{*}$ resonances makes this problem more complicated.

The theoretical approach sketched above has been applied to DEA to uracil and thymine [19], and also to the hydrogen loss in formic acid, glycine [25], and aminobutanoic acid [26]. In spite of the 
success of this approach, particularly in description of VFRs, and in spite of the excellent agreement with experiments on formic acid [27], several objections were made with regard to the existence of the $\sigma^{*}$ resonance in these systems. Indeed the standard Breit-Wigner parametrization of the scattering eigenphases did not allow for detection of a low-energy $\sigma^{*}$ resonance in formic acid [28], uracil [29-31] or glycine [32]. Our studies $[19,25]$ showed that the $\sigma^{*}$ resonances in these systems at the equilibrium nuclear geometry are extremely broad with the resonance width reaching $5-6 \mathrm{eV}$, therefore the resonance width actually exceeds the resonance energy. The time delay for this type of a resonance is insignificant, and the Breit-Wigner parametrization fails to produce any reasonable results, since the background phase shift varies with the same rate as the resonance phase shift, and sometimes, when there is a significant dipolar and polarization scattering, even faster.

It is reasonable to question the physical significance of such "superbroad" resonances. In fact the answer has been known for a long time since mid-80s when resonance vibrational excitation and DEA in collisions was studied and explained for hydrogen halides [33, 34]. More recently the state-of-the-art calculations for HF [35] were performed using the same ideas. In these systems the $\sigma^{*}$ shape resonance is barely visible, or sometimes not visible at all, in the elastic scattering. Nevertheless this resonance and its interaction with the dipole-supported state is crucial for producing threshold peaks in vibrational excitation and driving the DEA processes. In 2006 Michael Allan [36] noted that the same mechanism might be responsible for vibrational excitation of the $\mathrm{O}-\mathrm{H}$ stretch in formic acid. This conjecture was confirmed by DEA calculations [25] for this system.

Since the Breit-Wigner parametrization for "superbroad" resonances fail, as well as the parametrization based on the time-delay matrix [37], we need an alternative method for the decomposition of the scattering matrix into the resonance part and the background part. This is accomplished either by the Feshbach projection-operator approach [23] or the resonance R-matrix method [24]. In the latter the Breit-Wigner parametrization is carried out not for the scattering matrix, but for the R-matrix. Since the long-range part of the electron-molecule interaction is eliminated from the $\mathrm{R}$ matrix, it is not affected by the dipolar and polarization scattering whose contribution to the scattering matrix varies rapidly with energy.

As far as we are dealing with the equilibrium internuclear geometry, such a separation into the background and resonant part is ambiguous and does not represent an essential physics because of negligible time delay, as discussed above. However, when the nuclear geometry changes along the dissociation path, the resonance is stabilized and its width becomes small near the stabilization point where the temporary negative-ion state becomes bound. At this stage the resonant scattering becomes physically significant.

We conclude that the description of scattering at the equilibrium geometry in terms of a "superbroad" resonance is just a convenient mathematical tool allowing extrapolation of scattering parameters from the region close to the stabilization point, where the resonance description has a direct physical meaning, to the region of the equilibrium nuclear geometry. Physically it is very unlikely that the electron will be captured at the equilibrium geometry without autodetachment. However, at stretched geometries this process is becoming more likely, although the probability to find the molecule at a stretched geometry is decreasing with the deviation from the equilibrium. We have a competition between the Franck-Condon factor and the survival factor, well-known since the early work on the DEA process [38]. The magnitude of the DEA cross section depends critically on how quickly the stabilization occurs. The survival factor characterizes this process in the local approximation [38]. However, the long-range interaction causes threshold structures, VFRs and threshold cusps, which cannot be described by the local theory, therefore the survival factor argument can be used only qualitatively for systems with a strong long-range (dipolar and polarization) interactions. To illustrate this point, in the next section we present model calculations for uracil showing how DEA cross sections depend on the resonance width and the position of the stabilization point. 


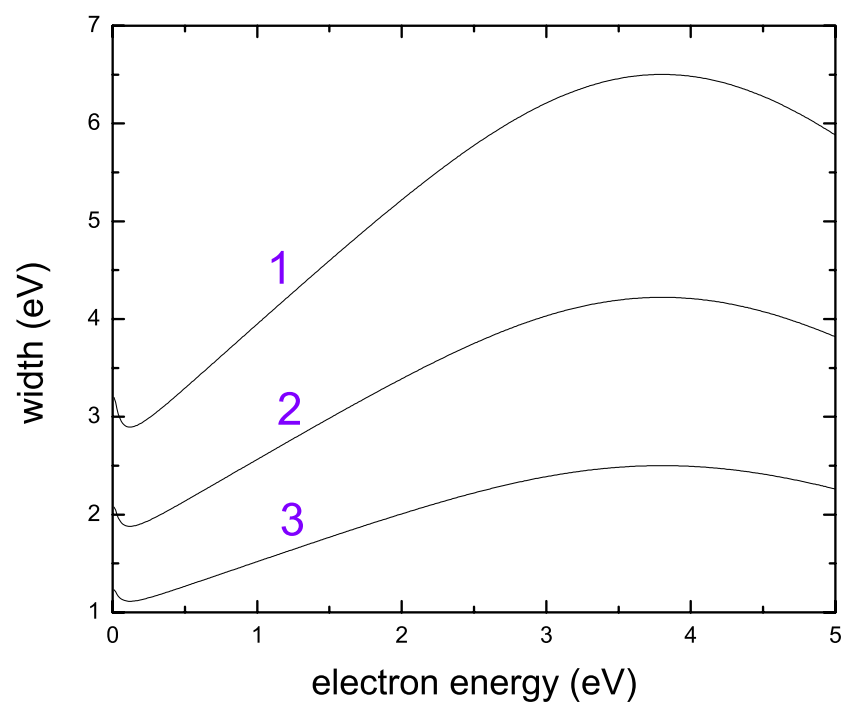

Figure 2. The resonance width as a function of energy for the equilibrium nuclear geometry in uracil. Curve 1 represents the original width. Curves 2 and 3 represent the widths reduced by factors 1.54 and 2.6 respectively.

\subsection{Model calculations for uracil}

We start with a model approach used in Ref. [19]. We assume one reaction coordinate roughly corresponding to the N1-H stretch. Then the process of $\mathrm{H}$ loss in thymine is not different from that in uracil, and the results can be applied to both molecules.

The resonance width used in calculations [19] is shown in Fig. 2. It was derived from the resonant R-matrix theory with the R-matrix surface amplitude and the position of the resonance pole fitted to reproduced the $a b$ initio width calculated using the Finite-Element Discrete Model [39].

The corresponding DEA cross section presented in Fig. 3 exhibit sharp peaks at the vibrational excitation threshold due to VFRs and threshold cusps. The resonance below the $v=3$ threshold is particularly pronounced. However, after averaging over the electron energy distribution with the width of $0.07 \mathrm{eV}$, typical for experiments, the peak value below the $v=3$ threshold is reduced to about $0.04 \times 10^{-16} \mathrm{~cm}^{2}$. To investigate the effect of the survival factor, we performed two series of model calculations. In the first, presented in Fig. 3, the resonance width was reduced by factors 1.54 and 2.6. In the latter case the scattering at the equilibrium nuclear geometry becomes more of the "classical" resonance scattering with a well-defined resonance width. The survival factor increases dramatically and the peak in the DEA cross section moves much closer to the resonance position $(3.13 \mathrm{eV})$. VFRs are not as pronounced in this case since the direct capture into the valence resonance state dominates.

In the second series of calculations we kept the resonance width unchanged but varied the anion potential curve in order to change the crossing (stabilization) point. The corresponding potential energy curves are shown in Fig. 4 where the solid (black) anion curve is the original curve used in calculations of the first series. It crosses the neutral curve at $\rho=0.703$ a.u. where $\rho$ is the N1-H separation relative to the equilibrium. The dashed (red) and dash-dotted (blue) curves cross the neutral at $\rho=0.732$ and 0.673 a.u. respectively.

The corresponding cross sections are presented Fig. 5. A strong increase in the absolute magnitude of the cross section is observed when the crossing point moves closer to the equilibrium internuclear separation. VFR at the $v=3$ threshold becomes much more pronounced. However, in contrast to the results of calculations of the first series, the position of the region where the DEA cross section 


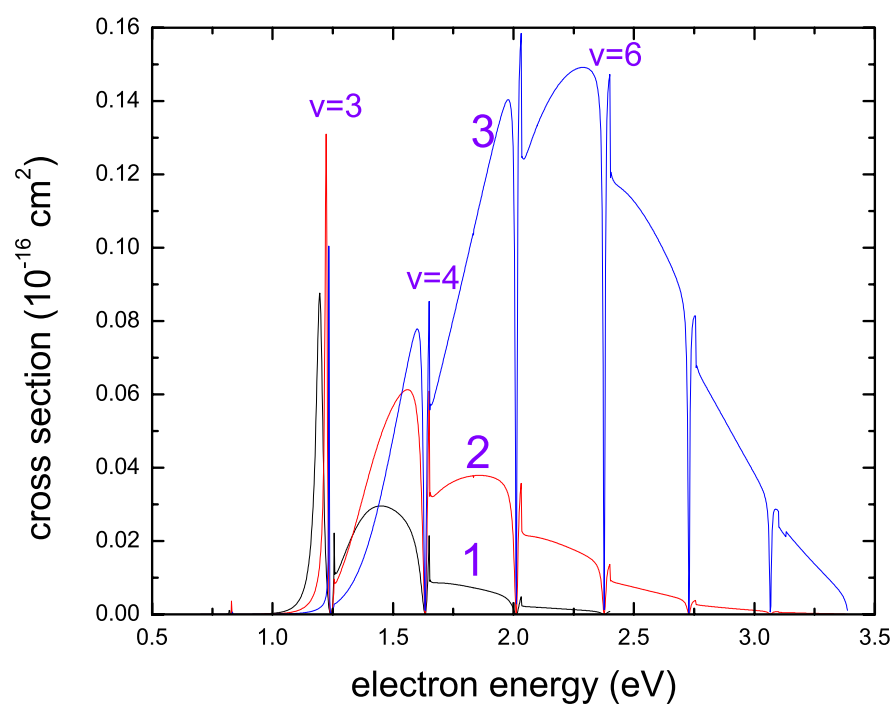

Figure 3. DEA cross sections for uracil. In calculations presented by curves 2 (red) and 3 (blue) the resonance width has been reduced by factors 1.54 and 2.6 respectively. The VFRs or threshold cusps are observed at the $v=3, \ldots, 8$ thresholds.

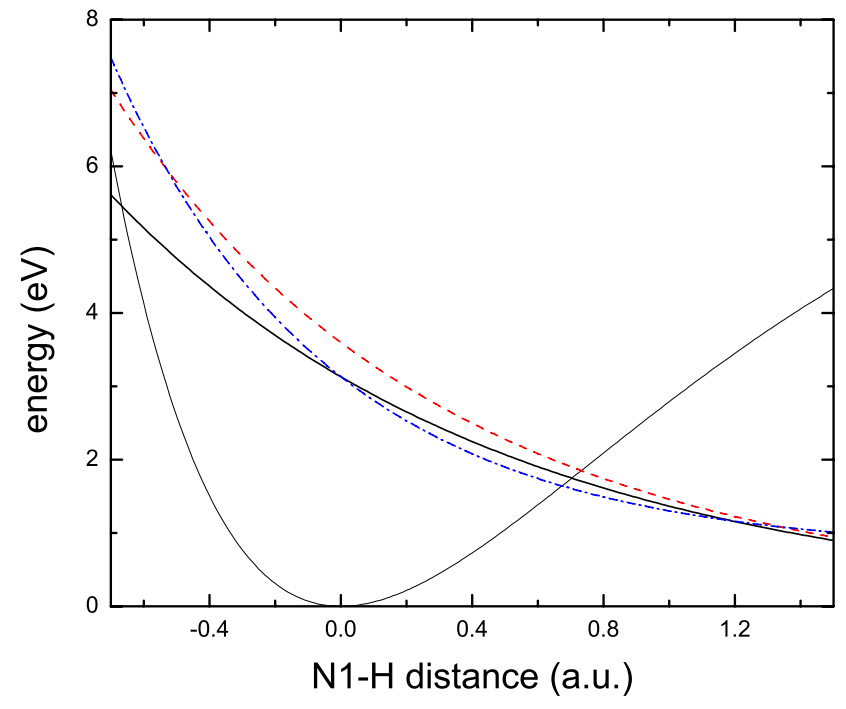

Figure 4. Potential energy curves showing dependence of energy on the $\mathrm{N} 1-\mathrm{H}$ separation relative to the equilibrium position. For solid (black) anion curve the crossing point is at 0.703 a.u., for dashed (red) curve at 0.732 a.u., and for dash-dotted (blue) curve at 0.673 a.u.

is substantial does not change at all. When the stabilization point is moved farther away from the equilibrium, the cross section becomes substantially smaller. We conclude that even with the "superbroad" resonance at the equilibrium nuclear geometry we can have a substantial DEA cross section if the resonance is stabilized fast when the geometry changes. 


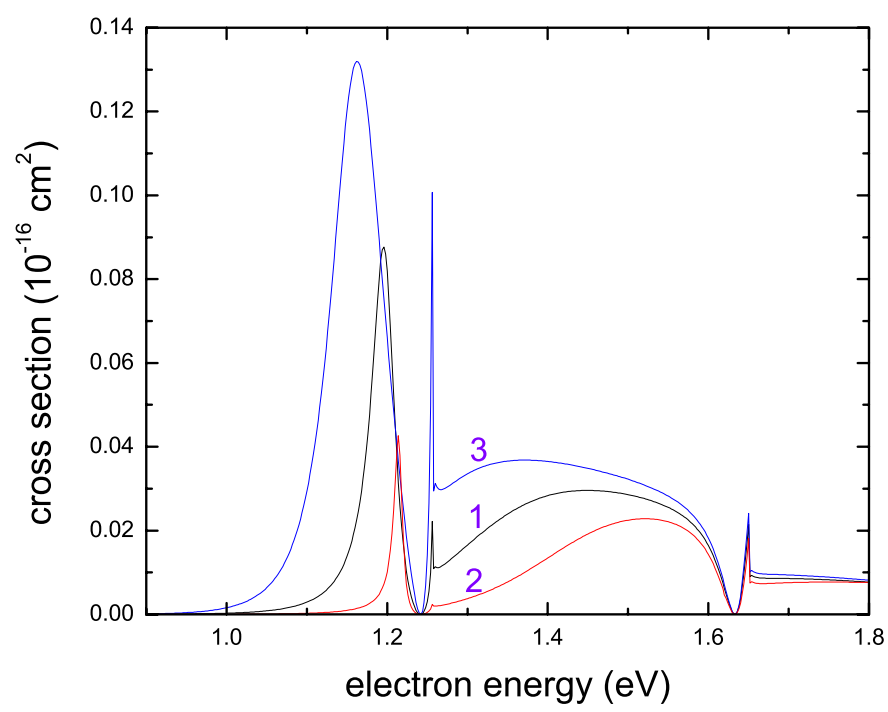

Figure 5. DEA cross sections for uracil. Calculations presented by lines 1 (black), 2 (red) and 3 (blue) correspond to the following anion curves given in Fig. 4: solid (black), dashed (red) and dash-dotted (blue).

\section{Multiple scattering theory}

In Ref. [40] we applied the multiple-scattering theory [14, 15, 41] to calculations of attachment amplitude in the presence of a water cluster. The expressions we used incorporated the multiple scattering effects only partly. Here we remove this deficiency by incorporating all scattering events self-consistently.

We assume that only one molecule captures the electron while others (the water molecules) act as rescatterers. In the spirit of the multiple-scattering theory [42], we represent the field of the cluster by a potential consisting of two types of regions defined by a set of nonoverlapping spheres. The potential within each sphere is equal to the potential (not necessarily spherically-symmetric) of the corresponding molecule, and we assume that the potential is zero outside the spheres. This approach is approximate, of course, since it neglects the long-range part of the potential due to each molecular unit. It is reasonable to neglect these long-range effects within the cluster. Outside the cluster these effects might be significant due to the effect of the net dipole moment and/or polarizability of the cluster. However, whereas these effects are important in elastic scattering, they should be insignificant in the electron attachment problem, since the resonance capture amplitude is dominated by a very few partial waves.

The asymptotic form of the wave function outside the sphere encompassing the molecule $n$ is

$$
\Psi\left(\mathbf{r}_{n}\right)=2 \pi i \sum_{L L^{\prime}} A_{L}^{n}\left[2 j_{l}\left(k r_{n}\right) \delta_{L L^{\prime}}+T_{L L^{\prime}}^{n} h_{l^{\prime}}^{(1)}\left(k r_{n}\right)\right] Y_{L^{\prime}}\left(\hat{\mathbf{r}}_{n}\right)
$$

where $\mathbf{r}_{n}$ is the electron position relative to the molecule $n, \mathbf{k}$ is the momentum of the incident electron, $L=(l, m), j_{l}$ and $h_{l}^{(1)}$ are spherical Bessel and Hankel functions, and $T_{L L^{\prime}}^{n}$ is the corresponding scattering matrix. The amplitudes $A_{L}^{n}$ are determined from the system of linear equations

$$
A_{L}^{n}(\mathbf{k})=e^{i \mathbf{k} \cdot \mathbf{R}_{n}} Y_{L}^{*}(\hat{\mathbf{k}})+\frac{1}{2} \sum_{n^{\prime} \neq n} \sum_{L_{1} L_{2} L_{2}^{\prime}} i^{l^{2}-l} A_{l_{2}}^{n^{\prime}}(\mathbf{k}) \tilde{F}_{m m_{1} m_{2}^{\prime}}^{l l_{1} l_{2}^{\prime}} T_{L_{2} L_{2}^{\prime}}^{n^{\prime}} h_{l_{1}}^{(1)}\left(R_{n n^{\prime}}\right) Y_{L_{1}}\left(\hat{\mathbf{R}}_{n n^{\prime}}\right)
$$


where $\mathbf{R}_{n}$ is the position of the center of mass relative to the origin, $\mathbf{R}_{n n^{\prime}}=\mathbf{R}_{n}-\mathbf{R}_{n^{\prime}}$, and

$$
\tilde{F}_{m_{1} m_{2} m_{3}}^{l_{1} l_{2} l_{3}}=i^{l_{1}+l_{2}-l}(-1)^{m}\left[4 \pi\left(2 l_{1}+1\right)\left(2 l_{2}+1\right)\left(2 l_{3}+1\right)\right]^{1 / 2}\left(\begin{array}{ccc}
l_{1} & l_{2} & l_{3} \\
0 & 0 & 0
\end{array}\right)\left(\begin{array}{ccc}
l_{1} & l_{2} & l_{3} \\
m_{1} & m_{2} & m_{3}
\end{array}\right) \text {. }
$$

The $\left(\begin{array}{c}\cdots \\ \ldots\end{array}\right)$ s represent Wigner $3-j$ symbols.

Equation (2) contains all amplitudes including the amplitude $A_{L}^{c}$ for the attaching molecule. After it is obtained, the capture amplitude $V^{(c)}$ is calculated as

$$
V^{(c)}(\mathbf{k})=\sum_{L} A_{L}^{c}(\mathbf{k}) V_{L}^{(0)}
$$

where $V_{L}^{(0)}$ is the partial capture amplitude for an isolated molecule related to the total capture amplitude $V^{(0)}(\mathbf{k})$ by the equation

$$
V^{(0)}(\mathbf{k})=\sum_{L} V_{L}^{(0)} Y_{L}^{*}(\hat{\mathbf{k}})
$$

The capture amplitude $V^{(c)}(\mathbf{k})$ can be rewritten in the following form convenient for calculations

$$
V^{(c)}(\mathbf{k})=\sum_{n L} C_{n L} e^{i \mathbf{k} \cdot \mathbf{R}_{n}} Y_{L}^{*}(\hat{\mathbf{k}})
$$

where

$$
C_{n L^{\prime}}=\sum_{L} V_{L}^{(0)}\left(M^{-1}\right)_{L L^{\prime}}^{c n}
$$

and the matrix $M$ is given by

$$
M_{L L^{\prime}}^{n n^{\prime}}=\delta_{L L^{\prime}}^{n n^{\prime}}-\frac{1}{2}\left(1-\delta_{n n^{\prime}}\right) i^{l^{\prime}-l} \sum_{L_{1} L_{2}} h_{l_{1}}^{(1)}\left(R_{n n^{\prime}}\right) Y_{L_{1}}\left(\hat{\mathbf{R}}_{n n^{\prime}}\right) \tilde{F}_{m m_{1} m_{2}}^{l l_{1} l_{2}} T_{L^{\prime} L_{2}}^{n} .
$$

Note that in the equations above the capture amplitude is dependent on internal nuclear coordinates $q$. Inclusion of this dependence is necessary for description of nuclear motion during the DEA process. First we calculate the resonance width $\Gamma(q, k)$

$$
\Gamma(q, k)=2 \pi \int\left|V^{(c)}(\mathbf{k}, q)\right|^{2} d \hat{\mathbf{k}} .
$$

For the calculation of the DEA cross section we solve an inhomogeneous Schrödinger equation with a nonlocal complex potential [23] which is constructed from the width function $\Gamma(q)$ and the shift function

$$
\Delta(q, k)=\frac{1}{2 \pi} \mathrm{P} \int_{0}^{\infty} d E^{\prime} \frac{\Gamma\left(q, k^{\prime}\right)}{E-E^{\prime}}
$$

where $E=k^{2} / 2$, and the symbol P stands for the principal value of the integral. The basic equation of the nonlocal complex potential theory is solved by the quasiclassical method [43, 44].

The scattering T-matrices for $\mathrm{H}_{2} \mathrm{O}$ were taken from the ab initio $\mathrm{R}$-matrix calculations as described in Ref. [40]. The ${ }^{2} A_{1}$ contribution to the elastic electron scattering by $\mathrm{CF}_{3} \mathrm{Cl}$ was calculated within the framework of the resonance R-matrix model [45] with some adjustments to get a better agreement with $a b$ initio calculations [46, 47].

In Fig. 6 we present the DEA cross sections for the $\mathrm{CF}_{3} \mathrm{Cl}$ molecule embedded in a water hexamer cluster for the geometry described in Ref. [40]. The cross section is very sensitive to the vertical attachment energy shift due to the cluster environment. This shift is called sometimes the "solvation energy." Because of some uncertainties in this quantity, we present results of two calculations: one with 


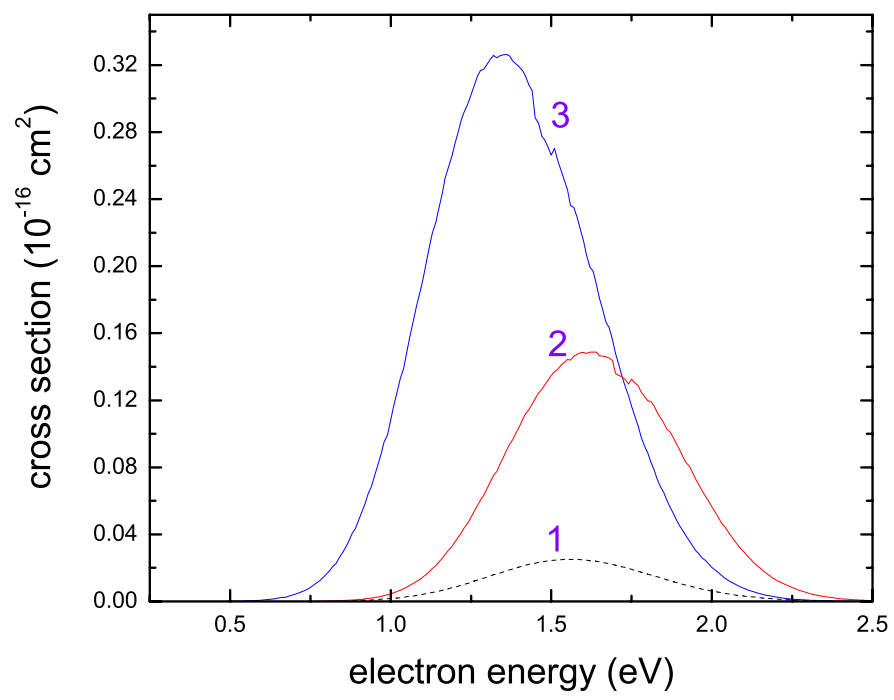

Figure 6. Cross section for DEA to the $\mathrm{CF}_{3} \mathrm{Cl}$ molecule. Dashed curve 1, isolated molecule. Curves 2 and 3: $\mathrm{CF}_{3} \mathrm{Cl}$ surrounded by the $\left(\mathrm{H}_{2} \mathrm{O}\right)_{6}$ cluster for the geometry of Ref. [40] for two solvation energies, 0 and $-0.2434 \mathrm{eV}$.

the zero shift (curve 2) and the other with the negative shift $-0.2434 \mathrm{eV}$. The present theory produces the cross section enhancement due to the cluster environment, as was already established in Ref. [40], although quantitatively results are somewhat different. An accurate calculation of the vertical attachment energy shifts is required for more reliable results for the DEA cross sections.

\section{Conclusion}

The major conclusion following from the recent studies of DEA to formic acid [25], uracil [19], aminobutanoic acid [26], and from the model calculations presented in the present paper, is that very broad $\sigma^{*}$ resonances play the crucial role in the hydrogen loss in these molecules due to low-energy electrons. Although these resonances are "superbroad" at the equilibrium nuclear geometry, they get stabilized fast when the bond is stretched.

Further development of the multiple scattering approach for DEA confirms the enhancement effect due to the cluster environment, although the DEA cross sections are very sensitive to the theory details, particularly to the vertical attachment energy shift due to the anion interaction with surrounding water molecules. For reliable quantitative results more research in this challenging field is necessary.

The author is grateful to G.A. Gallup, J. Gorfinkiel and Z. Masin for the fruitful collaboration. This work has been partly supported by the US National Science Foundation and by the Open University (UK) CEPSAR Research Centre.

\section{References}

[1] K. Aflatooni, G. A. Gallup, and P. D. Burrow, J. Phys. Chem. A 102, 6205 (1998)

[2] G. Hanel, B. Gstir, S. Denifl, P. Scheier, M. Probst, B. Farizon, M. Farizon, E. Illenberger, and T. D. Märk, Phys. Rev. Lett. 90, 188104 (2003)

[3] H. Abdoul-Carime, S. Gohlke, and E. Illenberger Phys. Rev. Lett. 92, 168103 (2004) 
[4] A. M. Scheer, K. Aflatooni, G. A. Gallup, and P. D. Burrow, Phys. Rev. Lett. 92, 068102 (2004)

[5] A. Pelc, W. Sailer, P. Scheier, M. Probst, N. J. Mason, E. Illenberger, and T. D. Märk, Chem. Phys. Lett. 361, 277 (2002)

[6] S. Gohlke, A. Rosa, E. Illenberger, F. Brüning, and M. A. Huels, J. Chem. Phys. 116, 10164 (2002)

[7] S. Ptasinska, S. Denifl, P. Candori, S. Matejcik, P. Scheier, and T. Märk, Chem. Phys. Lett. 403, 107 (2005)

[8] V. Vizcaino, P. Bartl, D. Gschliesser, S. E. Huber, M. Probst, T. D. Märk, P. Scheier, and S. Denifl, Chem. Phys. Chem. 12, 1272 (2011)

[9] T. Ito, S. C. Baker, C. D. Stickley, J. G. Peak, and M. J. Peak, Int. J. Radiat. Biol. 63, 289 (1993)

[10] B. D. Michael and P. O'Neill, Science 287, 1603 (2000)

[11] C.-R. Wang, J. Nguyen, and Q.-B. Lu, J. Am. Chem. Soc. 131, 11320 (2009)

[12] Q.-B. Lu and L. Sanche, Phys. Rev. B 63, 153403 (2001)

[13] J. Nguyen, Y. Ma, R. G. Bristow, D. A. Jaffray, and Q.-B. Lu, PNAS 108, 11778 (2011)

[14] D. Bouchiha, L. G. Caron, J. D. Gorfinkiel and L. Sanche, J. Phys. B 41, 045204 (2008)

[15] S. Caprasecca, J. D. Gorfinkiel, D. Bouchiha, and L. G. Caron, J. Phys. B 42, 095205 (2009)

[16] A. M. Scheer, C. Silvernail, J. A. Belot, K. Aflatooni, G. A. Gallup, and P. D. Burrow, Chem. Phys. Lett. 411, 46 (2005)

[17] P. D. Burrow, G. A. Gallup, A. M. Scheer, S. Denifl, S. Ptasinska, T. Märk, and P. Scheier, J. Chem. Phys. 124, 124310 (2006)

[18] S. Denifl, P. Sulzer, F. Zappa, S. Moser, B. Kräautler, O. Echt, D. K. Bohme, T. D. Märk, and P. Scheier, Int. J. Mass Spectrom. 277, 296 (2008)

[19] G. A. Gallup and I. I. Fabrikant, Phys. Rev. A 83, 012706 (2011)

[20] K. L. Stricklett, S. C. Chu, and P. D. Burrow, Chem. Phys. Lett. 131, 279 (1986)

[21] S. Ptasinska, S. Denifl, P. Scheier, E. Illenberger, and T. D. Märk, Angew. Chem. Int. Ed. 44, 6941 (2005)

[22] H. Hotop, M.-W. Ruf, M. Allan, and I. I. Fabrikant, Adv. At. Mol. Phys. 49, 85 (2003)

[23] W. Domcke, Phys. Rep. 208, 97 (1991)

[24] I. I. Fabrikant, Phys. Rev. A 43, 3478 (1991)

[25] G. A. Gallup, P. D. Burrow, and I. I. Fabrikant, Phys. Rev. A 79, 042701 (2009)

[26] V. Vizcaino, B. Puschnigg, S. E. Huber, M. Probst, I. I. Fabrikant, G. A. Gallup, E. Illenberger, P. Scheier, and S. Denifl, New J. Phys. 14, 043017 (2012)

[27] R. Janečková, D. Kubala, O. May, J. Fedor, and M. Allan, Phys. Rev. Lett. 111, 213201 (2013)

[28] C. S. Trevisan, A. E. Orel, and T. N. Rescigno, Phys. Rev. A 74, 042716 (2006)

[29] F. A. Gianturco, and R. R. Lucchese, J. Chem. Phys. 120, 7446 (2004)

[30] C.Winstead and V.McKoy, J. Chem. Phys. 125, 174304 (2006)

[31] A. Dora, J. Tennyson, L. Bryjko, and T. van Mourik, J. Chem. Phys. 130, 164307 (2009)

[32] J. S. dos Santos, R. F. da Costa, and M. T. do N. Varella, J. Chem. Phys. 136, 084307 (2012)

[33] W. Domcke and C. Mundel, J. Phys B 18, 4491 (1985)

[34] I. I. Fabrikant, S. A. Kalin, and A. K. Kazansky, J. Phys. B 25, 2885 (1992)

[35] M. Čižek, J. Horáček, M. Allan, I. I. Fabrikant, and W. Domcke, J. Phys. B 36, 2837 (2003)

[36] M. Allan, J. Phys. B 39, 2939 (2006)

[37] Z. Masin, private communication

[38] T. F. O’Malley, Phys. Rev. 150, 14 (1966)

[39] R. K. Nesbet, Phys. Rev. A 24, 1184 (1981)

[40] I. I. Fabrikant, S. Caprasecca, G. A. Gallup, and J. D. Gorfinkiel, J. Chem. Phys. 136, 184301 (2012)

[41] L. G. Caron and L. Sanche, Phys. Rev. Lett. 91, 113201 (2003) 


\section{EPJ Web of Conferences}

[42] D. Dill and J. L. Dehmer, J. Chem. Phys. 61, 692 (1974)

[43] S. A. Kalin and A. K. Kazansky, J. Phys. B 23, 4377 (1990)

[44] G. A. Gallup, Y. Xu, and I. I. Fabrikant, Phys. Rev. A 57, 2596 (1998)

[45] R. S. Wilde, G. A. Gallup, and I. I. Fabrikant, J. Phys. B 32, 663 (1999)

[46] T. Beyer, B. M. Nestmann, and S. D. Peyerimhoff, Chem. Phys. 255, 1 (2000)

[47] M. Tarana, K. Houfek, J. Horáček, and I. I. Fabrikant, Phys. Rev. A 84, 052717 (2011) 